\title{
Autisme et pratiques langagières: Analyse linguistique des interactions verbales entre éducateurs spécialisés et adultes avec T.S.A.
}

\author{
Sonia DeMartino ${ }^{1}$, Christina Romain², et Véronique Rey ${ }^{3}$ \\ ${ }^{1}$ Centre de Ressources Autisme, PACA \\ ${ }^{2}$ Aix-marseille Université, INSPE Aix-Marseille, LPL UMR7309 CNRS \\ ${ }^{3}$ Aix-marseille Université, INSPE Aix-Marseille, Centre de Recherche sur le Langage Orale et Ecrit \\ *Corresponding author : christina.romain@univ-amu.fr
}

\section{Résumé.}

Cette recherche propose de rendre compte à travers une approche linguistique (langage pragmatique et énonciation) des pratiques langagières réalisées entre adultes atteints de T.S.A. et professionnels. Ces professionnels sont des éducateurs spécialisés au sein d'une institution. Les situations linguistiques sont lors d'un trajet en voiture et lors d'un groupe de parole. L'analyse linguistique de ces échanges montre comment les acteurs participent aux actes de parole. La grille repose notamment sur les fonctions de la langue. Ces observations mettent en évidence les capacités des personnes atteintes de TSA dans des situations d'interaction verbale. Des pistes de travail sont proposées aux éducateurs pour considérer ces situations comme un levier de médiation thérapeutique.

\begin{abstract}
This research proposes to report through a linguistic approach (pragmatic language and enunciation) language practices carried out between adults with A.S.D. and professionals. These professionals are specialized educators within an institution. Language situations are during a car trip and during a talk group. The linguistic analysis of these exchanges shows how the actors participate in speech acts. The grid is based in particular on the language's functions. These observations highlight the abilities of people with ASD in verbal interaction situations. Working tracks are proposed to educators to consider these situations as a lever for therapeutic mediation.
\end{abstract}




\section{Introduction}

Le Trouble du Spectre de l'Autisme (T.S.A., DSM 5) se caractérise donc par des déficits persistants de la communication, des interactions sociales et des comportements ou des intérêts restreints. Ce trouble neurodéveloppemental apparaît dans l'enfance.

Les difficultés dans le domaine de la communication et des interactions sociales se manifestent par un manque d'intérêt à partager des activités, des émotions, à s'inscrire dans une réciprocité conversationnelle. Ces déficits génèrent des difficultés pour s'inscrire dans une relation avec un tiers. Les profils langagiers des individus présentant un T.S.A. sont très hétérogènes, allant de l'absence totale du développement du langage à une altération des pratiques langagières conversationnelles. Ces déficits ont des répercussions sur l'entourage, notamment les aidants familiaux et les accompagnants dans les instituts médico-sociaux qui peuvent se sentir démunis face aux difficultés relationnelles. Les altérations langagières d'une personne avec T.S.A. ne peuvent donc qu'altérer la relation établie avec l'entourage, voire remettre en question la pratique langagière elle-même du parent, de l'aidant ou du professionnel. Ceci fait écho à l'approche phénoménologique de Merleau-Ponty (1945). Merleau-Ponty présente l'activité langagière comme une extériorisation du sujet : au lieu de s'intéresser à la parole interne et individuelle, il s'agit de considérer l'activité langagière comme extérieure au sujet créant ainsi une obligation d'ouverture. Selon Merleau-Ponty, il s'agit d'une « expérience de la non-coïncidence avec soi ». C'est une activité au cœur de la socialisation du sujet. La situation énonciative donne sens, elle est riche de significations $a u$ fur et à mesure de ses emplois diversifiés.

Dans le quotidien, nous pratiquons nos actes de parole comme des évidences, sans conscience de la parole parlée. Les interactions verbales ne sont alors pas appréhendées comme un étayage de la construction des pratiques langagières singulières. Notre postulat est que, en situation professionnelle, ces actes de parole pourraient être un levier pour l'éducateur travaillant avec des personnes avec T.S.A. Nous rejoignons ainsi Merleau-Ponty qui appréhende la langue singulière comme le résultat de l'appropriation de nos interactions langagières avec autrui. Afin de rendre compte des contenus de ces interactions et de leur richesse potentielle, nous nous proposons de décrire, avec les outils de la linguistique, des interactions langagières entre professionnels accompagnants et personnes avec T.S.A. Cette approche est novatrice car, s'il existe de nombreux bilans de langage pour évaluer le code linguistique des personnes avec T.S.A., il n'y a pas d'analyses discursives de corpus portant sur des productions langagières écologiques, en langue française, entre personnes avec T.S.A. et personnes sans T.S.A.

La question est alors de savoir comment des éducateurs spécialisés échangent verbalement avec des adultes avec T.S.A. Pour répondre à cette question, il nous faut tout d'abord repérer comment la linguistique peut aborder ces phénomènes langagiers, puis rendre compte d'échanges langagiers ordinaires entre professionnels et adultes avec T.S.A. par une analyse discursive des corpus et enfin, à partir de ces observations et dans ce contexte théorique, proposer des pistes de travail pour le personnel et les aidants familiaux.

\section{La communication: acte de parole et interaction verbale, quelques points de repères}

Aborder par la linguistique, les dysfonctionnements des pratiques langagières chez les autistes nécessitent de faire un pas de côté et de repérer les langues comme des pratiques culturelles, participant à la socialisation des enfants (Rondal, 1983). Comme nos pratiques adultes ordinaires sont souvent automatisées et donc peu conscientes, nous avons voulu attirer l'attention du lecteur sur la nécessité de penser la mise en place de nos pratiques langagières comme le fruit de nos interactions sociales et culturelles. 
Nous rejoignons ainsi les propos de Troubetzkoy qui, dès 1964, indiquait :

" Chaque fois qu'un homme dit quelque chose à un autre homme, c'est un acte de parole. L'acte de parole est toujours concret; il a lieu à un endroit déterminé et à un moment déterminé. Il suppose : une personne déterminée qui parle, une personne déterminée à qui l'on parle et un état de choses déterminé auquel cet acte de parole se réfère. Par opposition à l'acte de parole, toujours unique, la langue est quelque chose de général et de constant. La langue existe dans la conscience de tous les membres de la communauté linguistique en cause et elle est le fondement d'innombrables actes de parole concrets. Mais la langue n'a d'autre raison d'être que de rendre possible l'acte de parole : elle n'existe qu'autant que les actes de parole concrets se réfèrent à elle, c'est-à-dire seulement dans la mesure où elle se réalise dans les actes de parole concrets. Sans actes de parole concrets, la langue n'existerait pas, de sorte que acte de parole et langue se supposent réciproquement » (Troubetzkoy, $1964: 1-3)$.

Ainsi, parler est une activité sociale qui met en jeu des pratiques langagières coénonciatives. Ces pratiques donnent à entendre la langue réalisée en acte de parole. L'interaction verbale (c'est-à-dire l'interaction en face à face) est définie par Goffman (1973 : 23) comme «l'influence réciproque que les participants exercent sur leurs actions respectives lorsqu'ils sont en présence physique immédiate les uns des autres ». Par conséquent, l'interaction induit une action réciproque des interlocuteurs. KerbratOrecchioni (2009: 103) définit quant à elle la négociation conversationnelle comme « tout processus interactionnel susceptible d'apparaitre dès lors qu'un différend surgit entre les interactants concernant tel ou tel aspect du fonctionnement de l'interaction, et ayant pour finalité de résorber ce différend afin de permettre la poursuite de l'échange ». La survenance du différend ou nœud de tension conduit à un conflit qui peut, s'il est exacerbé, conduire lui-même à la «mort de l'interaction » (Kerbrat-Orecchioni, 1992 : 48). Cette fin de l'interaction est traditionnellement définie comme la rupture définitive de toute influence réciproque (l'échange verbal cesse définitivement). Cependant des procédés de négociation conversationnelle peuvent être convoqués en ménageant par exemple la face de l'interlocuteur (cf. travaux de Brown et Levinson $(1978,1987)$ sur les procédés de politesse linguistique reposant sur des adoucisseurs).

\section{La dimension pragmatique du langage chez les individus atteints de T.S.A.}

Comme nous l'avons souligné par ailleurs (Rey et Romain, 2018), il nous parait nécessaire de montrer comment les relations interdiscursives de soin et d'accompagnement souvent informelles, entre les personnels d'instituts médico-sociaux et les adultes autistes, peuvent donner à voir les enjeux de l'interaction langagière et surtout comment elles interrogent certaines capacités à la fois discursives, interactionnelles et émotionnelles chez ces adultes atteints d'un TSA. Nous souhaitons analyser ces échanges interactionnels en nous éloignant d'une approche modulaire du langage (Lecours et al.,1987) ou encore évaluative via des classifications internationales (voir par exemple celle de l'American Psychiatric Association, 2000). Pour cela, nous nous inscrivons dans la lignée des travaux de Hupet (2006 : 89) qui souligne que les difficultés interactionnelles ne sont pas susceptibles d'être décrites par des tests procéduraux, car « les notions d'utilisation, d'usage, d'acte ou d'action et de contexte constituent le dénominateur commun à toutes les approches pragmatiques des phénomènes langagiers ». En effet, nous considérons les variations des pratiques énonciatives comme singulières et ancrées dans un lieu et un temps. De même, ces pratiques sont interactionnelles et impliquent autant les soignés eux-mêmes que les soignants-accompagnants de l'institut. L'interaction verbale reposant sur une influence réciproque des individus en présence selon Goffman (1973, 1974ab). Ainsi cet article est 
pour nous l'occasion de questionner la co-construction interactionnelle qui rend compte à la fois des rôles de chacun et de leurs influences respectives sur le devenir interactionnel de l'échange : quelle relation interactionnelle ? comment la relation interdiscursive peut-elle favoriser la mise en évidence de compétences communicationnelles chez les adultes autistes pris en charge dans des structures de soin ? Comment le professionnel peut-il être le « garant » de cette relation?

Les individus atteints d'un Trouble du Spectre Autiste sans déficience intellectuelle et avec une préservation du langage présentent généralement des troubles plus ou moins importants dans la dimension pragmatique du langage (Loukusa et Moilanen, 2009). S'ils peuvent participer à un échange interactionnel, ils se retrouvent néanmoins en difficulté dans sa gestion (Baron-Cohen, 1998 ; Rapin et Dunn, 2003) : ils appréhendent difficilement le principe de coopération conversationnelle (Grice, 1975) tout autant que celui des tours de parole (Ramberg et al. 1996). Ils ont tendance à méconnaître le thème interactionnel au profit de leur thème de prédilection (Baltaxe, 1977 ; Surian et al., 1996). Ils rendront difficilement compte de leurs états mentaux, ils éprouveront des difficultés à comprendre l'intentionnalité de leur interlocuteur (Baron-Cohen et al., 1985 ; Norbury et Bishop, 2002), et ils se heurteront à des difficultés à inférer, tout autant qu'à traiter l'information implicite, par exemple lors d'actes de langage indirects (Rey \& al. 2012). Enfin, ils éprouvent des difficultés à identifier les éléments du contexte de la situation d'énonciation (Ivanko et Pexman, 2003 ; Cummings, 2005). Ainsi, Tager-Flusberg (1994, 1999, 2000) a montré l'existence d'une altération de l'usage pragmatique du langage chez ces individus dès leur plus jeune enfance. Cette altération des troubles verbaux d'ordre pragmatique (Rapin et al., 2003) a alors tendance à s'accroître avec le développement de la verbalisation (TagerFlusberg et al., 2005). Ces individus utilisent le langage dans des buts pas ou peu sociaux (Boucher, 2003). C'est pourquoi on parle le plus souvent de trouble de la communication (Frith, 1989 ; Frith et Happé, 1994). Lord et Paul (1997) soulignent quant à eux le manque de cohésion conversationnelle et la faiblesse des initiatives communicationnelles. Rapin et al. (2003) soulignent en ce sens l'existence d'une pauvreté de l'expression et de la formulation plus générale du discours.

La visée de notre article est finalement multiple car elle interpelle à la fois le fonctionnement interactionnel de la relation de soin mais aussi son appréhension/analyse interactionnelle par le soignant-accompagnant. En effet, notre étude vise à observer comment des capacités interactionnelles chez l'adulte autiste sont susceptibles d'émerger en des circonstances interactionnelles non prévues. Elles pourraient être susceptibles à tout le moins d'éveiller l'attention des soignants-accompagnants et de les encourager à stimuler l'échange.

Si certaines études montrent comment certains sujets peuvent percevoir «que nous voulons tirer ses mots en dehors de lui afin que, de cette façon terrible, ils deviennent les nôtres et ne lui appartiennent plus » (Alvarez, 2007), Alvarez soulignant d'ailleurs l'importance d'utiliser la «bonne longueur d'onde » (1999) afin d'interagir avec ces sujets, il pourrait être particulièrement utile pour ces soignants-accompagnants de repérer ces « ondes » positives pour solliciter l'interaction verbale. Cela d'autant plus que DanonBoileau et Morel (2007), étudiant le discours oral d'un sujet adulte autiste dans sa multimodalité, montrent comment ce dernier marque son émotion mais de manière non conforme par rapport à un adulte proto-typique. Ces auteurs vont jusqu'à poser que cette émotion rencontre une difficulté voire une impossibilité à se communiquer. Les auteurs poursuivent en mettant en avant que les marques d'expression des affects sont souvent non comprises par les interactants car ces marques choisies ne sont pas identifiées comme telles. Ils concluent enfin leur propos en regrettant la simplicité des discours qui consistent à 
conclure sur un défaut de théorie de l'esprit chez ces adultes autistes. Un recours « en conscience " aux pratiques langagières pourrait alors avoir un effet positif en termes d'accompagnement par ces personnels dans la verbalisation des émotions des adultes autistes mais pas seulement. Nous allons étudier deux échanges qui ont eu lieu, pour l'un à l'occasion d'une régulation d'un dysfonctionnement interactionnel a posteriori, lors d'une réunion de groupe, et pour l'autre à l'occasion d'une communication informelle en transport en voiture à l'occasion de laquelle l'adulte autiste se livre. Nous observerons ces échanges dans une perspective d'analyse pragmatique. Cela dans la lignée d'études récentes portant sur la relation de soin montrant comment le lien entre praticien et patient peut être tissé afin de favoriser l'échange en le mettant au service de la relation de soin/clinique (Boudart, 2018; Grosjean, Hezlaoui-Hamelin et Pedersen, 2018 ; Gülich, 2018 ; Rey et Romain, 2018).

Par étude pragmatique, nous entendons l'étude du contenu et de l'usage de la pratique interactionnelle du langage dans sa dimension communicationnelle. La pragmatique verbale réfère au fonctionnement de l'usage communicatif du langage. Ce fonctionnement renvoie aux règles de l'alternance des tours de parole, des initiatives et des réponses, du maintien d'une conversation organisée (rester sur un thème donné), et l'usage stylistique des modes d'expression comme le choix des mots qui doivent être appropriés à la situation sociale. Ce fonctionnement renvoie aussi à une expression et une compréhension (traitement de l'implicite et des processus d'inférence), à la cohérence sémantique et à la cohésion syntaxique. Par ailleurs, on ne saurait oublier que la pragmatique a une composante non verbale que nous ne traiterons pas dans cet article mais qui ne manquera pas d'être traitée par ailleurs. Surtout, l'approche pragmatique repose sur un contenu tout comme sur un usage social du langage reposant sur des habiletés conversationnelles, au titre desquelles on trouve : une communication authentique et intentionnelle, le recours aux fonctions de communication du langage (établir et maintenir un échange, produire des actes de langage de la vie courante et quotidienne (Austin, 1962 ; Searle, 1972), etc), respecter les règles conversationnelles (gestion des tours de parole, cohésion syntaxique et cohérence sémantique, etc.). À partir des travaux de Bühler (1934/2009) qui a attribué trois fonctions au langage liées à la personne et à son accomplissement, Jakobson (1973) a décrit plusieurs fonctions du langage. Parmi les fonctions de la communication, la fonction conative est orientée sur le destinataire par l'émetteur qui est alors en recherche d'influence sur ce destinataire. Ainsi les actes de langage développés dans la théorie d'Austin sont ici particulièrement éclairant tout autant que le recours ou pas à des procédés de ménagement des faces selon la théorique de la politesse linguistique. Nous citerons ici la pratique de la politesse linguistique qui consiste à ménager la face de l'autre tout autant que sa propre face lors $\mathrm{du}$ fonctionnement interactionnel mais aussi lors d'un dysfonctionnement interactionnel (Brown et Levinson, 1978, 1987 ; Kerbrat-Orecchioni, 2009).

Les outils d'analyse interactionnelle et également les outils du côté de l'analyse des habiletés socio-émotionnelles nous permettront de rendre compte d'une compréhension tout autant que d'une expression émotionnelle (Hobson, 1993; Trevarthen, 2007). D'une manière générale, nous considérons qu'une remédiation consiste à une prise en charge des troubles de la communication reposant sur un travail portant sur le développement des fonctions de communication et sur l'adaptation à l'environnement des modalités de communication. Mais parmi les habiletés sociales, on va également trouver le principe de pertinence (Sperber et Wilson, 1989), les lois du discours (Ducrot, 1979) et les maximes de de la conversation (Grice, 1975). Lakoff, quant à lui (1973), s'intéresse aux postulats de la conversation, tandis que Sacks, Schegloff et Jefferson (1974) rendent compte de la structuration de l'échange en termes tours de parole. 


\section{Les enjeux des pratiques langagières co-énonciatives: une étude de cas}

Notre étude repose sur l'interaction à visée conversationnelle que constitue l'échange entre des résidents avec T.S.A. et des accompagnants. Dans la perspective de l'analyse des interactions verbales, nous avons étudié le contenu des échanges dans deux situations (groupe de parole, trajet en voiture).

\subsection{Questionnement de l'échange singulier pour une pragmatique singulière}

Nous avons conduit une étude reposant sur l'analyse d'un corpus constitué de vidéos rendant compte de groupes de parole entre praticiens/accompagnants et autistes au sein d'une association accueillant depuis plus de quarante ans des résidents autistes, présentant des troubles du spectre de l'autisme et des enfants polyhandicapés. L'association vise à permettre à ses résidents de faire « émerger leur potentialité en installant le travail au centre de leur projet de vie ». Par conséquent, l'interaction verbale, la relation interdiscursive qu'elle permet de mettre en place, et plus largement la relation interpersonnelle entre résidents et accompagnants se trouvent être au cœur du fonctionnement de l'association. Il nous a été possible d'accéder à des vidéos, fournis par les accompagnants, rendant compte de la vie quotidienne des résidents.

Les corpus ont été analysés du point de vue du discours en interaction (KerbratOrecchioni, 1996, 2009). Notre objectif a été de mieux comprendre comment agir sur l'interaction verbale afin de favoriser sa richesse (la stimulation et la prise en compte de la parole du résident autiste) tout autant que son apaisement (la gestion et l'anticipation des moments de montée en tension verbale voire de rupture interactionnellei) dans un contexte d'accompagnement et de soins quotidiens, permanents. Pour cela, nous avons convoqué des outils de l'analyse de discours, de l'analyse conversationnelle et plus largement de la linguistique interactionnnelle (du discours en interaction). Notre point d'encrage théorique a visé à décrire à la fois :

-la relation interdiscursive conversationnelle : tours de parole, chevauchement, interruption, structuration de l'interaction etc.

- la relation interpersonnelle : à travers la gestion (ou pas) des ruptures interactionnelles potentielles (gestion des nœuds de tension dans cette interaction spécifique), et à travers la stimulation (ou pas) de la parole dans la dynamique interactionnelle pathologique.

Nous avons élaboré une analyse prenant en compte les indicateurs suivants :

-habiletés conversationnelles: fonctions de la communication, initiatives communicationnelles, principe de pertinence, lois du discours/maximes conversationnelles, actes de langage, gestion des tours de parole, cohésion syntaxique et cohérence sémantique ;

-habiletés socio-émotionnelles : compréhension et expression des émotions ; procédés de ménagement des faces (politesse linguistique); états mentaux (théorie de l'esprit) et intentionnalité

\subsection{Présentation des résultats}

Deux situations langagières sont décrites ci-dessous. Il y a systématiquement au moins une personne avec T.S.A. et un éducateur spécialisé. Toutes les personnes (le personnel et les personnes avec T.S.A.) ont plus de 30 ans. Les situations enregistrées ont été choisies par le personnel et les enregistrements ont été réalisés grâce à eux. 
Exemple 1 Situation d'interaction langagière lors d'une situation nécessitant une pratique langagière spécifique (groupe de parole).

Un incident a eu lieu la veille au soir : des cris ont éclatés dans le foyer suite à une dispute entre deux résidents $\mathrm{S} v s \mathrm{~F}$ Lors du groupe de parole, cet incident est repris par l'accompagnant (P1) en présence des deux résidents.

Extrait de l'échange :

P1 : alors apparemment hier soir euh/ puisque tu parles de F/ et c'est vrai que + dans les quelques jours où tu as été mal/ $\mathrm{F}$ il a été pendant euh aussi d'un grand soutien/ parce que c'est lui qui a un petit peu appeler/ quand tu as eu des difficultés

Arlette : C'est vrai

P1 : Hein/ d'accord/ S.

$\mathrm{S}:$ Ah oui j'ai fait les

P1 : Et hier soir/ apparemment/ il y a eu un incident

$\mathrm{S}$ : Alors j'ai fait/ je lui ai dit que c'était bon/ son omelette + mais : je lui ai dit qu'il $\mathrm{y}$ avait des coquilles dedans

P1 : alors n'oublie pas/ S./ il faut que tu fasses attention/ des fois/ à la façon dont tu t'exprimes/ d'accord

$\mathrm{S}: \underline{\text { d'accord }}$

$\mathrm{P} 1$ : tu as une façon de parler + qui des fois est un petit peu trop:: directe/ qui peut être gênante/ pour quelqu'un qui a fait l'effort de préparer le repas/ ça peut arriver de laisser des coquilles/ moi j'ai le souvenir que des fois dans la salade il restait des chenilles aussi/ quand tu l'avais nettoyée/ c'est des choses qui peuvent arriver fréquemment/ donc on peut dire/ au ben tiens j'ai eu une coquille/ et puis voilà

$\mathrm{S}:$ oui $<\ldots$ ? $>$ mais $\mathrm{j}$ 'ai dit qu'il était très bon/ ton omelette

(Plusieurs bruits de voix)

$\mathrm{S}$ (en direction de $\mathrm{F}$ ) : Et puis ça t'inquiète ces histoires de pain (F hoche la tête) moi j'avais faim/ et j'ai mangé du pain

P2 : Au moment du repas?

$\mathrm{S}:$ ben oui

F : Et toi tu me l'as dit après/ au repas qu'on mange de, bon/ ben qu'on n'a pas mangé/ et toi t'as mangé les deux bouts

$\mathrm{S}$ : ben oui mais j'avais faim

P1 : Non mais S + c'est des choses que l'on a évoqués aussi (soupire)/ c'est une tranche de pain par repas/ hein c'est pas la question d'avoir/ il y avait autre chose à manger + que le pain (hochement de la tête en direction de $\mathrm{S}$ )

P3 : Vous vous rappelez que vous sortez le pain/ du congélateur/ le pain que vous allez manger pour le repas du congélateur/ vous ne laissez pas un pain entier dehors hein/ parce que sinon vous avez tendance + à le grignoter/ et à manger tout le pain

\section{$\mathrm{A}:<\ldots ?>$}

$\mathrm{P} 1$ : Après F peut-être/ quand y a des:: des soucis comme ça/ si tu te sens:: un peu agressé par la façon dont te parle $\mathrm{S}+/$ ça serait bien que tu essaies de pas te mettre en colère tout de suite + hein de pouvoir lui dire ben écoute/ la façon dont tu me parles/ ça me convient pas/ et si tu n'es pas bien/ tu peux peut-être en parler avec nous/ hein mais peutêtre éviter de se mettre en colère/ de crier/ parce qu'après du coup c'est A qui est mal

A : oui c'est vrai j'ai j'ai la colique calme

P1 : voilà/ donc si vous pouviez arriver à communiquer/ de façon/ un petit peu plus

$\mathrm{S}:$ Ben quand je rentre/ après je m'excusais hein $\mathrm{A}$

A : c'est pas ça c'est pas ça $<\ldots$ ? $>$ après j'ai appelé $<\ldots$ ? $>$ il est venu $<\ldots$ ? $>$

$\mathrm{P} 1$ : non mais tu as bien fait/ si tu étais dérangée par le comportement/ tu as bien fait Arlette

$\mathrm{A}:<\ldots ?>$ 
P1 : A chacun de faire un petit effort/ pour parler de façon un peu plus calme

$A$ : Mais là je ne suis pas énervée $C$

$\mathrm{P} 1$ : Non mais je parlais à $\mathrm{F} /$ de dire les choses peut-être un peu plus calmement

On observe tout d'abord que S intervient dans l'échange conversationnel alors que sa prise de parole était demandée seulement dans l'implicite de celle de P1 «Et hier soir/ apparemment/ il y a eu un incident». S intervient alors afin d'expliquer la situation rencontrée la veille, d'en donner plus spécifiquement sa propre version. La parole de $\mathrm{S}$ repose sur ce qu'elle a dit, sur les faits. Notons ici que le choix des faits qui vont être rapportés et articulés par un «mais » rendent bien compte d'une dimension réflexive quant à la situation vécue par S. Pourtant cette réflexivité reste dans l'implicite, il n'y a pas de contextualisation de cette situation. Ainsi S initie son intervention par la valorisation de la face de F (le plat préparé par F était bon : son omelette était bonne) puis elle informe sur la présence de morceaux de coquille d'œuf dans le plat préparé. S. recourt ainsi à la fonction référentielle (elle informe sur ce qui s'est passé) ainsi qu'à la fonction expressive et à la fonction conative à travers le «mais» qui oppose. Ainsi, dans son propos, dans sa restitution des événements, il y a une trace d'argumentation qui met l'accent à la fois sur l'expression de son " avis » et sur ce qui a «tendu » l'échange afin d'éclaircir la situation de tension. $\mathrm{S}$ est consciente que $\mathrm{F}$ a mal pris son constat de la présence de coquilles dans l'omelette, pourtant, dans le même temps, elle ménage sa face en soulignant que l'omelette était bonne. Par sa prise de parole et son contenu, elle informe sur le ménagement dont elle a fait preuve mais sans le rendre explicite en tant que tel. P1 lui rappelle alors qu'elle ne doit pas oublier de faire attention à sa façon de parler. $\mathrm{S}$ répond par un « d'accord », par là elle convoque la fonction à la fois expressive (elle donne son avis) et la fonction conative (elle s'inscrit en cohérence avec le contenu de la parole de P1). P1 poursuit en soulignant que $\mathrm{S}$ a une façon de parler « qui des fois est un petit peu trop:: directe ». Ce à quoi $\mathrm{S}$, après avoir laissé P1 terminé son intervention, répond par un « oui $<\ldots$ ? > mais j'ai dit qu'il était très bon/ ton omelette ». S recourt à nouveau à la fonction conative (elle cherche à convaincre qu'elle n'était pas dans la menace... elle a valorisé sa camarade car elle a dit que son omelette était bonne, elle met en avant ce point « mais... omelette »), par ailleurs elle poursuit son inscription en cohérence avec son interlocuteur « oui ». On observe que $\mathrm{S}$. recourt de ce fait à la fonction expressive puisqu'elle dit quelque chose relevant de son propre positionnement, elle ne recherche pas la polémique, mais l'argumentation. Cependant, S n'explicite pas tout cela, ne contextualise pas son propos, ne rappelle pas les fait en les commentant, en introduisant une dimension explicitement réflexive.

Dans une perspective d'analyse interactionnelle, on observe tout à la fois une pertinence de son intervention, un respect des tours de parole, une cohérence sémantique et une cohésion syntaxique de son propos en adéquation avec le contenu de l'échange initié par P1. Surtout, on observe aussi la prise en compte du ménagement de la face de F par $\mathrm{S}$ qui est verbalisée par cette dernière. Cependant, cette verbalisation n'est pas suffisamment contextualisée et aucun recourt à des éléments rendant compte d'une argumentation structurée n'est présent en nombre suffisant pour donner un contenu argumenté à son propos. La forme n'est pas celle qui est courante et peu passer presque inaperçue. S comprend l'inférence de P1 : son propos a été trop menaçant. Elle lui répond par un « oui mais » qui initie un argument soulignant le ménagement dont elle a fait preuve et donc ce sur quoi elle a nécessairement prêté attention afin de «faire attention au contenu de sa parole ». Pourtant P1 ne le relève pas. Probablement car P1 ne retient que l'opposition formulée par le «mais» sans s'attarder sur le contenu sémantique et l'implicite l'accompagnant en termes d'adoucisseur et de ménagement des faces. P1 retient l'acte de langage assertif visant une potentielle opposition polémique (contre discours) alors que cet acte de langage interactionnellement vise davantage l'explicitation argumentée (discours 
alternatif). Autrement dit, il apparait ici que cette formulation est globalement non conforme par rapport à un adulte proto-typique qui mettrait plus d'explicitation et plus de ménagement dans le contenu de son propos (comme le fait P1 d'ailleurs au début de l'échange notamment) comme par exemple «j'ai pourtant dit que ton omelette était bonne, cependant il est vrai qu'il y avait des coquilles à l'intérieure et cela était dommage ». La forme adoptée par S peut ne pas être suffisamment explicite et finalement peut être non comprise par les interactants : F étant émotionnellement atteint par la critique négative faite à son encontre et $\mathrm{P} 1$ étant parasité par le comportement antérieur auquel il réfère pour parler de la façon de parler de S. Il est par ailleurs important de souligner ici que S identifie bien l'intentionnalité de P1 puisqu'elle tente d'expliquer qu'elle a soigné le contenu de sa parole. $\mathrm{S}$ va aussi jusqu'à prendre en compte $\mathrm{F}$ et va faire émerger une cause extérieure à la formulation langagière choisie qui expliquerait la dispute qui a éclatée «et puis ça t'inquiète ces histoires de pains ». On apprend alors que $S$ a mangé la portion de pain destinée à $\mathrm{F}$. En effet, $\mathrm{F}$ lui reproche alors d'avoir mangé tout le pain et $\mathrm{S}$ répond seulement en constatant qu'elle avait faim. Elle ne ménage pas l'action qu'elle a produite mais est capable, sans le rendre explicite, de faire un lien entre une réaction exacerbée de $\mathrm{F}$ à son reproche ménagé et une seconde action. On constate alors que, du côté de $\mathrm{S}$, la théorie de l'esprit et l'intentionnalité imputée à autrui son actif ici. Il est alors significatif d'observer que l'échange en présence de l'accompagnant est dense. $\mathrm{S}$ a recouru à la fonction conative à l'adresse de $\mathrm{F}$ (elle prend en compte les émotions de F) et à elle satisfait à la fonction expressive en expliquant le choix qu'elle a fait de manger le morceau de pain qui ne lui était pas destiné.

On observe que $\mathrm{S}$ utilise et traite à travers son propos, de la politesse interactionnelle (ménagement interactionnelle de la face). La politesse linguistique est mise en mot en termes de valorisation de son propos. Par ailleurs, si S ne verbalise pas explicitement le lien entre l'émotion ressentie par $\mathrm{F}$ (qui a vu mangé son morceau de pain) et sa réaction au reproche formulé par S (présence de morceaux de coquille d'œufs dans l'omelette), elle le réalise néanmoins implicitement en formulant «et puis ça t'inquiète ces histoires de pain/ moi j'avais faim/ et j'ai mangé du pain »). Cependant, à nouveau, on observe une dimension implicite dans l'explicitation de la scène vécue la veille. Le propos de $\mathrm{S}$ se focalise sur les faits (omelette réussie, présence de coquilles, faim, bout de pain mangé) mais leur organisation syntaxique rend compte d'un sens (à la fois dans une perspective sémantique, mais aussi linéaire, chronologique) prenant en compte l'émotion et l'intentionnalité d'autrui et de soi. S cherche tout au long de l'échange à expliquer/justifier son comportement et elle le fait en intégrant la dimension émotionnelle qu'elle perçoit/comprend chez l'autre. Elle rend également compte d'une conscience du ménagement nécessaire de la face de l'autre. Tout autant qu'elle elle s'inscrit dans la validation du positionnement de P1 (« oui », «d'accord ») et le respect des tours de parole et de la thématique engagée. L'accompagnant P1 ne semble pas observer ces éléments, il ne voit pas les habiletés conversationnelles et socio-émotionnelles dont fait preuve $\mathrm{S}$. Probablement par le manque d'explicitation contextuelle lié à un défaut du respect des maximes de la conversation: si S en informant, disant ce qu'elle pense être vrai, est pertinente par rapport à l'attente interactionnelle et est sans ambiguïté, claire et ordonnée dans son propos, elle ne l'est pourtant pas de façon suffisante, elle ne recourt que aux faits et les articule entre eux sans une mise en mots explicite. Cela, contrairement à un adulte proto-typique, contrairement finalement à $\mathrm{P} 1$ qui rappelle des règles explicites de fonctionnement de l'interaction verbale attendue dans le centre. P1 ne semble pas repérer la richesse du propos de $\mathrm{S}$.

Au-delà du dispositif lui-même (le groupe de parole), on observe d'emblée l'intérêt que lui portent les résidents. Ils sont calmes. On « voit » l'attention partagée, le corps est à l'écoute. Le groupe de parole, en tant qu'outil de résolution des conflits mais aussi en tant 
qu'outil ritualisé où la langue a un rôle central à jouer, nous intéresse tout particulièrement.

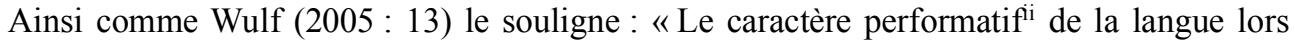
des actions rituelles a une importance cruciale. ». Le groupe de parole fonctionne alors comme rituel-outil de résolution des crises. Wulf (2005: 17-18) insiste sur le fait qu'en contexte de menace, de danger, les rituels sont un moyen de se «comprendre sur le plan relationnel grâce à une forme de mise à distance de l'objet du conflit permettant de résoudre le conflit en cours et ainsi de conduire les individus à intégrer à nouveau la communauté interactionnelle. Il s'avère donc tout particulièrement pertinent de s'intéresser à la structuration de ce rituel et à ce qui peut le faire dysfonctionner en termes de rupture interactionnelle. Plus généralement encore, Amigues, et Zerbato-Poudou (2000) rajoutent qu'un rituel est efficace seulement s'il garantit à chacun de s'impliquer.

Exemple 2: Situation d'interaction langagière lors d'une situation ne nécessitant pas une pratique langagière spécifique (trajet en voiture).

La conversation a lieu en voiture entre la résidente $(\mathrm{S})$ et l'accompagnante (PY). PY vient de prendre $\mathrm{S}$ en voiture pour la reconduire à l'appartement. Le thème de la conversation n'a aucun rapport sémantique avec l'activité (déplacement lors d'un trajet en voiture). La conversation commence sur la situation que $\mathrm{S}$ vient de vivre (visite chez un orthodontiste). C'est S qui initie la conversation. Celle-ci est constitutive de plusieurs thématiques en termes d'échanges :

l'a

$\mathrm{S}:$ Bon ben demain/ c'est c'est $<\ldots$ ? > son appareil là $/<\ldots$ ? > c'est le docteur X qui

PY : D'accord/ donc là ton projet rendez-vous c'est quand là

S : J'irai soit jeudi/ soit vendredi

$\mathrm{PY}$ : oui c'est ça/ là je l'ai pas en tête

$\mathrm{S}:$ mon appareil

PY : d'accord +++ ça va/ ça s'est bien passé ?

$\mathrm{S}$ : ouais

PY : qu'est-ce qu'il t'a fait/ là donc

$\mathrm{S}:$ ben rien/ il m'a fait les $<\ldots$ ? $>$ / et::

$\mathrm{PY}$ : et tu as essayé le le nouveau?

$\mathrm{S}:$ non pas encore

PY : non pas encore/ d'accord

$S$ : il m'a fait les empreintes cette fois

PY : d'accord/ ok ++ bon ben c'est bien/ parce que je sais pas si tu allais penser à prendre les sacs (lorsque $\mathrm{S}$ est entrée dans la voiture, elle a doNné un gros sac à PY)

$\mathrm{S}:$ j’y ai pensé

PY : c'est bien/ tu as anticipé

$\mathrm{S}:$ merci pour le pyjama (grand sourire et regard en direction de PY)

$\mathrm{PY}$ : ben donc/ i i tu l'as essayé ? (S regarde PY)

$\mathrm{S}$ : ouais (S regarde toujours PY et lui sourit)

PY : bon ben c'est c'est c'est vingt euros/ il coute vingt euros (S regarde toujours PY et lui sourit)

$\mathrm{S}:$ bon (S regarde PY et lui sourit toujours)

PY : on fera un ticket/ si il te convient + tu vas le garder ( $\mathrm{S}$ sourit toujours mais détourne le regard, elle regarde devant elle) +++ ça c'est bien passé hier soir ?

$\mathrm{S}:$ oui ++ Arlette a fait les tomates et les poivrons $/<\ldots$ ? $>$

PY : elle était un peu dure

S : par contre la pâte elle l'a fait trop cuire (S regarde en face d'elle)

$\mathrm{PY}$ : elle était un peu dure?

$\mathrm{S}$ : elle a un peu fait trop cuire (S regarde en direction de PY mais pas son visage) 
PY : d'accord

S : c'était bon/ mais vu la cuisson/ si la pâte elle se casse/ (elle regarde devant elle) c'est un peu trop cuit (S regarde PY et sourit)

$\mathrm{PY}$ : elle était bonne quand même

S : oui

PY : bon + c'est ça qui compte hein/ tu sais des fois on fait des des (PY est interrompue par $\mathrm{S}$ )

$\mathrm{S}:$ des erreurs

PY : manger/ même si c'est un peu trop cuit/ mais c'est un très bon très bon gout + en tout cas elle avait l'air belle

(Dix secondes s'écoulent)

$\mathrm{S}$ bon je (S baille) ben j'attendrai un peu pour m'acheter un $<\ldots$ ? $>$ (Elle regarde devant elle) + parce que (elle regarde en face d'elle puis elle regarde PY et sourit de façon accentuée)

PY : ben euh on verra + comment ça peut::: si là t'en voit un/ on peut regarder

$\mathrm{S}:$ Oui + parce que là tout à l'heure $<\ldots$ ? $>$

$\mathrm{PY}$ : Ben bien sûr

$\mathrm{S}$ : Parce que rester enfermé là

PY : Surtout qu'il fait beau là

(Dix secondes s'écoulent, $\mathrm{S}$ baille à nouveau)

$\mathrm{PY}$ : ben dis donc/ t'es fatiguée là

$\mathrm{S}$ : oui/ je me suis levée à 8 heures et demi/ ce matin

$\mathrm{PY}$ : ben ça va/ c'est raisonnable ++ tu t'es pas couchée trop tard?

$\mathrm{S}:$ non

$\mathrm{PY}: \mathrm{t}$ 'as regardé quelque chose à la télé/ hier soir

$\mathrm{S}:$ non

PY : non (quelques secondes s'écoulent) t'as repris un petit peu le coloriage

$S$ : un peu

PY : ou pas encore

$\mathrm{S}$ : mais presque

(Silence, $\mathrm{S}$ regarde devant elle et a les bras croisés)

Ce second exemple interactionnel vise à rendre compte de la propension d'initiations d'échanges qu'un adulte TSA est susceptible de réaliser dans une situation singulière donnée : une accompagnante assure le transport en voiture d'un adulte atteint d'un TSA du centre à son rendez-vous médical et le ramène ensuite au centre. Afin que ces échanges soient favorisés et surtout développés, nourris en termes d'interventions des accompagnants, il faudrait que ces derniers les repèrent. Très souvent l'accompagnant recherche des thèmes conversationnels, comme cela est le cas dans cet exemple, or il s'avère que dans le même temps l'adulte atteint d'un TSA initie de lui-même des thèmes qui ne sont pas ou peu repris par l'accompagnant. Il s'agirait alors pour ce dernier de développer une nouvelle forme d'attention langagière, de les percevoir « en conscience » en quelque sorte, afin d'entrer en échange conversationnel avec le résident « initiateur langagier ».

Si un premier échange lié à la situation de déplacement en voiture (rendez-vous médical) est initié par la résidente qui va spontanément commencer à raconter son rendezvous médical, on observe que l'accompagnante ne va pas hésiter à changer de thème pour parler des sacs : «d'accord/ ok ++ bon ben c'est bien/ parce que je sais pas si tu allais penser à prendre les sacs ». S initie alors un nouvel échange sur le thème du pyjama «merci pour le pyjama » avec marqueur de politesse linguistique («merci »). L'accompagnante sera à nouveau à l'initiative du changement de thème suivant (le déroulé de la soirée précédente) tout comme pour celle du quatrième thème (la fatigue supposée de la résidente) 
et cela alors même que la résidente reviendra sur le thème du pyjama «bon je ben j'attendrai un peu pour m'en acheter un $\langle\ldots$ ? $>»$. On observe ensuite que, lors de ce quatrième échange, les interventions de $\mathrm{S}$ sont réduites : elle intervient systématiquement lorsque son tour de parole arrive mais ne le développe pas davantage. Après la première intervention (fonction référentielle « oui/ je me suis levée à 8 heures et demi/ ce matin »), le contenu est très réduit («non », « un peu », « mais presque »). Puis la conversation s'éteint de longues secondes. De même, la résidente regarde régulièrement l'accompagnante au début et au milieu de la conversation, mais lors du quatrième échange elle regarde devant elle, les bras croisés (un peu comme si la conversation arrivait à son terme).

L'accompagnante est centrée sur la fonction référentielle du langage, change rapidement de sujet et ne parvient pas à rebondir sur les sujets qui sont initiés par la résidente (le pyjama, le rendez-vous médical, le plat cuisiné de la veille). Dans ces échanges, les émotions ne sont jamais évoquées (émotions dans le cabinet médical, lié à l'achat/besoin du pyjama, à la qualité d'un plat, à la fatigue, etc.). On dirait que ce sont des thématiques qui sont systématiquement évitées alors que les résidents eux-mêmes n'hésitent pas à les convoquer.

A l'issue de cette analyse, on aura dégagé trois points interactionnels spécifiques :

- Le trajet en voiture est pertinent dans le contexte observé où le résident à des informations à transmettre à l'accompagnant (qui n'était pas avec lui).

- Il est pertinent lorsque le résident lui-même l'initie (il est probable que ce type de contexte soit facilitant pour lui).

- Il est moins pertinent pour l'intervenant. Ce dernier semble en difficulté et génère des stimulations essentiellement référentielles qui s'éteignent rapidement.

\section{Synthèse des observations préliminaires et proposition}

A l'issue de l'analyse de notre corpus, il apparait que le personnel accompagnant sous-expose souvent les résidents à un langage adressé autre que référentiel et s'enferment dans les thématiques qu'ils ont eux-mêmes choisies et qu'ils n'hésitent pas à modifier de façon successive comme dans l'exemple 2. Le personnel se focalise régulièrement en production sur la fonction référentielle. Il laisse peu de place à la dimension expressive des résidents qui pourtant semble être susceptible d'expliciter les choix en termes de fonction conative du langage de ces derniers et plus largement un accès à la dimension réflexive. Les stratégies langagières des accompagnants sont le plus souvent non coopérative et donc, dans une certaine mesure, inefficace interactionnellement en termes d'influence réciproque favorable à une coopération et à l'élaboration d'un à venir commun au sein d'une même structure médico-sociale. Dans le même sens, on aura aussi observé que les accompagnants ne donnent pas la parole aux résidents pour parler de leurs émotions (les expliquer, etc.) alors que certains sont prêts à en parler, à les décrire. De fait, les échanges étudiés rendent compte d'une grande difficulté à lier affect et parole alors que les résidents sont susceptibles d'y trouver un intérêt. Paradoxalement, on aura observé des accompagnants qui reprochent aux résidents leurs émotions ou des émotions négatives qu'ils ont procurées à autrui, dans le même temps où ils leur refusent leur propre expression émotionnelle. Ainsi, il s'avère que le groupe de parole en termes d'attention partagée, conjointe, de coopération et de négociation est extrêmement pertinent : ils sont en actes de parole. Mais, lorsque les résidents et les accompagnants sont en « action » (trajet en voiture), cela s'avère beaucoup plus difficile car les actes de parole ne sont pas pratiqués comme des actes thérapeutiques.

$\mathrm{Au}$ contraire, les résidents quant à eux montrent à plusieurs reprises, à travers les échanges étudiés, qu'ils peuvent «parler de » et même analyser les situations conflictuelles 
à la fois dans une dimension réflexive et dans une dimension émotionnelle et intentionnelle. La mise en mots permettrait pourtant de toucher les codes sociaux là où le contexte seul ne suffit pas. En réception, les résidents pourraient ainsi percevoir/recevoir du langage social. On observe à que les résidents seraient capables de partager des pratiques énonciatives (l'un d'entre eux reconnait avoir blessé l'autre par ses propos, etc.) et de parvenir à accéder à la négociation lors de situations interactionnelles délicates, potentiellement conflictuelles.

Nos principales observations montrent donc que :

1. Les résidents sont « capables » de participer aux actes de parole à partir de faits les concernant directement ou indirectement dans un passé proche. Cependant les personnels ne les encouragent pas à parler « sur» ou « de » leurs émotions.

2. L'analyse conversationnelle confirme l'existence d'échanges structurés et cohérents en termes d'habiletés conversationnelles (ex : les tours de parole sont respectés, tout comme la cohérence sémantique et la cohésion syntaxique, productions d'actes de langage), mais aussi d'habiletés socio-émotionnelles (compréhension et expression des émotions, ménagement des faces, prise en compte des états mentaux et de l'intentionnalité).

3. L'importance du fonctionnement ritualisé qui organise la parole, la distribue, donne le temps à chacun de s'exprimer etc. Les résidents ne sont n'y agités ni agressifs, lors du groupe de parole.

4. Les moments de tension sont récurrents et peuvent passer inaperçus ou ne pas être traités comme tels par les accompagnants : ces derniers ont tendance à interrompre les thématiques interactionnelles des résidents dans leurs échanges spontanés.

A la lumière de ces observations, plusieurs pistes peuvent être proposées.

\subsection{Identifier et maitriser le dialogue singulier}

Ce serait par le dialogue singulier entre un autiste et un accompagnant que pourrait se transmettre un savoir-faire et un savoir-être. Au cours de ces échanges, l'accompagnant écoute, observe l'autiste. Intuitivement, il élabore une empathie avec l'autiste prenant en compte tout ce qu'il sait de l'autiste, son environnement social, familial, ses capacités. Il n'existe pas d'autiste «hors-sol». En même temps, l'accompagnant rassemble ses connaissances techniques et culturelles. La disponibilité de l'accompagnant, sa façon d'être, sa gestion de la parole échangée, quelquefois ses silences sont tout aussi nécessaires. Difficile, redoutable exercice tant sur le plan intellectuel, culturel qu'affectif. De son côté, l'autiste est venu avec une demande mais aussi avec tous ses problèmes. Il peut communiquer, il fait confiance, mais en même temps, il suit son propre cheminement interactionnel, sans concession. Il faut le motiver interactionnellement, c'est-à-dire choisir le bon moment et le bon sujet, pour pouvoir interagir positivement avec lui, au-delà d'un seul tour de parole. Le dialogue singulier est alors une rencontre entre deux personnes, entre celui qui maitrise les codes de l'interaction verbale sociale et celui qui ne les maitrise pas de la même manière, pas selon la prévisibilité de la personne sans T.S.A. L'efficience du dialogue singulier dépend de la relation que deux personnes sont capables de nouer, de développer entre eux, de leur langage commun construit au fil des échanges. Il comporte nécessairement une charge émotionnelle car, ce n'est plus seulement un échange ponctuel mais des rapports qui vont s'enrichir à travers les événements de la vie. L'accompagnant s'initie au monde de la personne avec T.S.A. et cette dernière apprend de son côté la nature et l'étendue de la qualité de l'échange avec son accompagnant voire de l'enseignement qu'il peut recevoir de son accompagnant. Mais cela se ferait d'autant plus que l'accompagnant fait preuve d'une vigilance interactionnelle spécifique.

\subsection{Dialogue singulier et tensions verbales}


En cas de tensions verbales, le dialogue singulier n'est plus alors uniquement dialogue mais prend une dominante émotive. L'accompagnant est bien parfois dans la situation où il doit réagir pour passer une phase critique qui empêcherait la personne avec T.S.A. de communiquer. L'accompagnant a un pouvoir de communication et de stimulation à la communication qu'il doit comprendre, identifier, maitriser pour l'utiliser au bon moment, en fonction des besoins et des sollicitations de la personne avec T.S.A. L'accompagnant doit alors maintenir la vigilance pour ne pas perdre celui qui peut lui faire confiance pour le mener sur le chemin des actes de parole ordinaires. Cette vigilance se réalise en explicitant les propos et en réduisant le traitement implicite des actes de langage. Autrement dit, l'accompagnant doit veiller à connaitre l'autiste, son histoire, ses envies, ses intérêts, etc. pour mieux orienter la gestion des tensions verbales.

\subsection{Des pratiques langagières en conscience}

On pourrait ainsi proposer comme objectif pour les accompagnants de ne pas faire parler « longtemps » les résidents mais de dépouiller une thématique qui les stimule sous tous les aspects (référentiels, relationnels, émotionnels, narration et procédure, etc). Cette thématique pourrait être repérée et intervenir uniquement en contexte, dans un échange singulier. Il serait intéressant de bien les identifier (tout ce qu'il est possible de proposer comme questionnement, comme tour de parole aux résidents) et ensuite aussi et dans le même temps de repérer le sujet qui leur convient le mieux au moment de la conversation (même s'il est polémique : ce serait l'occasion de transmettre et de mettre en mots les codes sociaux, pour qu'ils puissent les reconnaitre, discuter dessus, etc.).

Ainsi, au-delà des moments de groupes de parole, des moments tels que, par exemple, la préparation du repas du soir où les résidents se retrouvent avec les accompagnants sont donc propices aux échanges langagiers qui, bien qu'étant relativement courts et faisant intervenir des interlocuteurs successifs, sont l'occasion de transmission langagière, énonciative, etc... Il parait donc essentiel que les résidents soient conscients de la présence de ces occasions d'échanges langagiers (contexte propice), mais surtout de l'importance de les repérer et de les pratiquer en conscience (à la fois en termes de reformulation, d'aide à la production lexicale, et d'un point de vue conversationnel -prendre soin de répondre, de relancer, et de laisser du temps (un temps minimum) pour l'expression du résident).

La voix entre alors en jeu et devient la pièce maitresse, le fondement d'une prise en charge non pas des autistes eux-mêmes mais des accompagnants afin de favoriser l'entrée en communication, l'interaction avec les autistes. Construire une relation interpersonnelle et surtout interdiscursive favorable à la prise de parole et à sa production par l'autiste serait singulière. Lorsque ces sujets émergent de la parole de l'autiste, comme cela peut être le cas lors des groupes de parole, l'accompagnant doit favoriser l'échange autour de ce sujet. S'il apparait que l'autiste présente un certain retard de maturité sociale et de raisonnement social (difficulté par exemple à reconnaitre ce qui peut mettre autrui dans l'embarras), une empathie relativement immature ou à tout le moins un délai de traitement des informations sociales ralenti, il semble néanmoins que dans certaines situations interactionnelles ils soient capables d'identifier certaines émotions à certains moments qu'ils ont pu provoquer ou s'en défendre d'ailleurs aussi (en tout cas il parait intéressant de pouvoir mettre en mots et à distance les émotions éprouvées ou à tout le moins les contextes correspondants).

\section{Conclusion}

L'intérêt de notre étude a été de repérer les moments propices aux échanges langagiers qui donneraient lieu à des échanges de points de vue, au sein duquel il serait possible de voir émerger notamment la théorie de l'esprit chez l'adulte atteint d'autisme 
mais aussi les principes de coopération linguistique. A partir de notre corpus, nous avons donc cherché à identifier ces moments et à mieux comprendre comment les accompagnants peuvent les favoriser mais aussi et surtout comment ils peuvent les reconnaitre et les favoriser grâce à leur propre production verbale. Mais l'intérêt de notre étude réside aussi dans le fait d'avoir pu montrer que les résidents adultes atteints d'autisme de nos exemples sont dotés d'habiletés communicationnelles et d'habiletés socio-émotionnelles susceptibles d'émerger lors d'interactions verbales informelles (trajet en voiture) ou formelles (groupe de parole).

À la vue de nos résultats, la personne avec T.S.A. serait capable d'accéder aux pratiques communicationnelles et aux intentions d'autrui dans le cas d'une rupture interactionnelle. Autrement dit, l'autiste pourrait être capable d'identifier certaines émotions émanant d'autrui dans un contexte interactionnel verbal en tension (premier exemple) ou encore être prêt à interagir et à partager des émotions et des faits personnels et finalement y renoncer du fait du peu d'impact de ses interventions chez l'accompagnant (exemple 2). L'accompagnant, qui chercherait par ce positionnement à éviter un sujet de discorde ou un sujet qui pourrait lui apparaître comme sans intérêt risquerait alors de manquer une occasion de stimuler l'interaction verbale avec l'adulte atteint d'autisme.

Les résultats de notre étude invitent donc à poursuivre ces explorations langagières afin de mieux cerner les capacités non seulement des personnes avec T.S.A. mais aussi des accompagnants en situation d'interaction thérapeutique.

\section{Références bibliographiques}

Alvarez, A. et Reid, S. (1999). Autism and Personnality : Findings from the Tavistock Autism Workshop. Londres: Routledge.

Alvarez, A. (2007). Trouver la bonne longueur d'ondes : les outils de communication avec les enfants autistes. In B. Touati et al., Langage, voix et parole dans l'autisme (pp. 238-260). Paris: Presses Universitaires de France.

Amigues, R., et Zerbato-Poudou, M. (2000). Comment l'enfant devient élève. Paris: Retz. Benveniste, E. (1966). Problèmes de linguistique générale, T.1 et 2. Paris: Gallimard.

Association, A. P. (2000). Diagnostic and Statistical Manual of Mental disorders. Text revision (DSM -IV-TR) (4ème édition ed.). Washington, DC: American Psychiatric Association.

Austin, J. L. (1962). How to do Things with Words. Oxford: Oxford University Press

Baltaxe, C. (1977). Pragmatics deficits in the language of autistic adolescents. Journal of Pediatric Psychology. 2. p. 176-180.

Baron-Cohen, S. (1998). La cécité mentale, un essai sur l'autisme et la théorie de l'esprit. Grenoble, Presses Universitaires de Grenoble.

Baron-Cohen, S., Leslie, A., \& Frith, U. (1985). Does the autistic child have "a theory of mind"?, Cognition.21 (1), p. 37-46.

Boucher, J. (2003). Language development in autism. Congress International Series, $1254,247-253$.

Boudart, I. (2018). Bilan orthophonique : quand plusieurs langues s'invitent. In K. Ploog, S. MarianiRousset et S. Equoy Hutin (Dir.), Emmêler et démêler la parole. Approche pluridisciplinaire de la relation de soin, 205-212, Besançon : Presse Universitaires de Franche-Comté.

Bühler, K. (1934/2009). Théorie du langage. La fonction représentationnelle du langage. Marseille: Didier Samain et Janette Friedrich. Agone.

Cummings, L. (2005). Pragmatics : A muldidisciplinary perspective. Edinburgh, UK : Edinburgh University Press.

Danon-Boileau, L. et Morel, M.-A. (2007). Approche linguistique du discours autistique : quelques remarques. In B. Touati et al., Langage, voix et parole dans l'autisme (pp. 335-340). Paris: Presses Universitaires de France.

Ducrot, O. (1979). Les lois du discours. Langue française, 42, 21-33.

Fusarolli, R. et Tysen, K. (2016). Investigating conversationnal dynamics : Interactive alignment, interpersonal synergy, and collective task performance. Cognitive Science, 40, 145-171. 
Frith, U. (1989). A new look at language and communication in autism. British Journal of Disorders of Communication, 24, 123-150.

Frith, U. et Happé, F. (1994). Language and communication in the autistic disorders. Philosophical Transactions of the Royal Society, series B, 346, 97-104.

Fusaroli, R., Lambrechts, A., Yarrow, K., Maras, K. et Gaigg, S. (2016). Conversational voice pattern in adult English speakers with ASD. Paper presented at the IMFAR 2016, Baltimore, United States.

Goffman, E. (1973). La mise en scène de la vie quotidienne. Tomes $1 \&$ 2. Paris : Editions de Minuit.

Grice, H.-P. (1975). Logic and conversation. In P. Cole \& J. L. E. Morgan (Eds.), Syntax and Semantics : Speech Acts (pp. 41-58). New York: Academic Press.

Grosjean, L. Hezlaoui-Hamelin, S. et Pedersen, L. (2018). L'interaction de soin à la croisée des regards : quand la sociologie et les sciences du langage se rencontrent. In K. Ploog, S. MarianiRousset et S. Equoy Hutin (Dir.), Emmêler et démêler la parole. Approche pluridisciplinaire de la relation de soin, 283-299, Besançon : Presse Universitaires de Franche-Comté.

Grossman, R.B., Bemis, R.H, Skwerer, D.P, Tager-Flusberg, H. (2010). Lexical and affective prosody in children with high-functionning autism. Journal of Speech Language and Hearing Research, 53, 778-793.

Gülich, E. (2018). Analyser la parole pour établir un diagnostic : perspectives du travail pluridisciplinaire entre médecins et linguistes. In K. Ploog, S. Mariani-Rousset et S. Equoy Hutin (Dir.), Emmêler et démêler la parole. Approche pluridisciplinaire de la relation de soin, 187-203, Besançon : Presse Universitaires de Franche-Comté.

Hobson, P. (1993). Autism and Development of Mind. Hove: Laurence Erlbaum.

Hupet, M. (2006). Bilan de la compétence pragmatique. In E. Estienne \& B. E. Priérart (Eds.), Bilan de langage et de voix. Paris : Masson.

Ivanko, S.L. \& Pexman, P.M. (2003). Context incongruity and irony processing. Discource Processes, 35, p. 241-279.

Jakobson, R. (1973). Essais de linguistique générale, Rapports internes et externes du langage, T.2. Paris: Les éditions de Minuit.

Kerbrat-Orecchioni, C. (2009). Le discours en interaction. Paris : Armand Colin.

Kerbrat-Orecchioni, C. (1996). La conversation. Seuil, Paris.

Kerbrat-Orecchioni, C. (1995). Les interactions verbales, t. 1. Paris: A. Colin.

Kerbrat-Orecchioni, C. (1992). Les interactions verbales, t. 2. Paris: A. Colin.

Lakoff, R. (1973). The logic of politeness ; or, Minding your p's and qs. The Ninth Regional meeting of the Chicago Linguistic Society

Lakoff, R. (1977). What You Can Do With Words : Politeness, Pragmatics, and Performatives. In A. Rogers, B. Wall \& J.-P. E. Murphy (Eds.), Proceedings of the Texas Conference on Performatives, Presupposition, and Implicatures. Arlington: Center for Applied Linguistics.

Lecours, A. R., Dumais, C., \& Tainturier, M. J. (1987). Les aphasies. In M. J. E. Botez (Ed.), Neuropsychologie Clinique et neurologie du comportement (pp. 307-324). Montréal et Masson: Presses Universitaires de Montréal.

Lord, C. et Paul, R. (1997). Language and communication in autism. In D. J. Cohen et F. R. Volkmar (Eds), Handbook of Autism and Developmental Disorders (pp. 195225). New York : John Wiley \& Sons.

Loukusa, S., Moilanen, I. (2009). Pragmatic inference abilities in individuals with Asperger syndrome or high-functioning autism. A review. Research in Autism Spectrum Disorders, 3, p. 890-904.

Merleau-Ponty, M. (1945). Phénoménologie de la perception. Paris: Gallimard.

Norbury, C.F. \& Bishop, D.V.M. (2002). Inferentiel processing and story recall in children with communication problems : A comparision of specific language impairment, pragmatic language impairment and high-functioning autism. International Journal of Language and Communication Disorders, 37, p. 227-251.

Pickett, E., Pullara, O., O’Grady,J. et Gordon, B. et (2009). Speech acquisition in older nonverbal individuals with autism : A review of features, methods, and prognosis. Cognitive and Behavioral Neurology, 22, 1-21.

Ramberg, C., Ehlers, S., Nydén, A., Johansson, M., \& Gillberg, C. (1996). Language and pragmatic functions in school-age children on the autism spectrum. European Journal of Disorders of Communication, 31, 387-414.

Rapin, I. et Dunn, M. (2003). Update on the language disorders of individuals on the autistic 
spectrum. Brain \& Development, 25, p.166-172.

Rapin, I., Dunn, M. et Allen D. A. (2003). Developmental language disorders. In J. Segalowitz (Ed.), Handbook of Neuropsychology (pp. 593-630). Amsterdam: Elsevier Science.

Rey, V., Demartino, S., Girardot, A.M., Poinso, P. (2012). Premier étalonnage pour évaluer un déficit dans le traitement de la métaphore chez des enfants présentant un syndrome d'Asperger, ANAE, 24, 117, (tome II), 208-214.

Rey, V. et Romain, C. (2018). Les pratiques langagières au coeur de la relation de soin.

In K. Ploog, S. Mariani-Rousset et S. Equoy Hutin (Dir.), Emmêler et démêler la parole. Approche pluridisciplinaire de la relation de soin, 169-185, Besançon : Presse Universitaires de Franche-Comté.

Rondal, J. A. (1983). L'interaction adulte-enfant et la construction du langage. Bruxelles: Mardaga.

Sacks, H., Schegloff, E., \& Jefferson, C. (1974). A simplest systematics for the organization of turntalking in conversation. Language, 50(4), 696-735.

Searle, J. (1969). Speech Acts. Cambridge: Cambridge University Press.

Sperber, D. et Wilson, D. (1989). La pertinence : communication et cognition. Paris: Editions de Minuit.

Surian, L., Baron-Cohen, S. \& Van Der Lely, H. (1996). Are children with autism deaf so Grice an maxims ? » Cognitive Neuropsychology, 1 (1), 55-71.

Tager-Flusberg H. (1994), Dissociations in form and function in the acquisition of language by autistic children. In H. Tager-Flusberg (Ed.), Constraints on Language Acquisition : Studies of Atypical Children (pp. 175-194). Hillsdale (NJ): Erlbaum.

Tager-Flusberg, H. (1999). Autisme infantile. In J. A. Rondal et X. Séron (Eds.), Troubles du langage (pp. 641-657). Liège: Mardaga.

Tager-Flusberg, H. (2000). Understanding the language and communicative impairments in autism. International Review of Research in Mental Retardation, 23, 185-205.

Tager-Flusberg H., Paul R., Lord C. (2005). Language and communication in Autism. In F. Volkmar, R. Paul, A. Klin et D. Cohen (Eds), Handbook of Autism and Pervasive Developmental Disorders (pp. 335-364). New-Jersey: John Wiley and Sons.

Trevarthen, C. (2017). Autisme et langage. In B. Touati et al., Langage, voix et parole dans l'autisme (pp. 217-237). Paris: Presses Universitaires de France.

Troubetzkoy, N. (1964[1939]). Principes de Phonologie, traduit de l'allemand par J.Cantineau. Paris: Klincksieck.

Wulf, C. (2005). Rituels. Performativité et dynamique des pratiques sociales. Hermès, 43, 9-20.

\footnotetext{
i On entend par rupture interactionnelle, un refus de débat ou un refus d'entendre ou de prendre en considération la parole de l'autiste par l'accompagnant.

ii Un énoncé performatif permet la réalisation d'une action par le fait même de son énonciation comme par exemple les propos d'un maire «je vous marie» (référence http://www.cnrtl.fr/definition/performatif).
} 